\title{
Antiviral Therapy Reduces Mortality in Hepatocellular Carcinoma Patients with Low-Level Hepatitis B Viremia
}

\section{Xinhui Wang (D) \\ Xiaoli Liu \\ Peng Wang \\ Lihua Yu (D) \\ Fengna Yan \\ Huiwen Yan (D) \\ Dongdong Zhou \\ Zhiyun Yang (D)}

Center of Integrative Medicine, Beijing Ditan Hospital, Capital Medical University, Beijing, 1000I5, People's Republic of China
Correspondence: Zhiyun Yang Center of Integrative Medicine, Beijing Ditan Hospital, Capital Medical University, Beijing, I000I5, People's Republic of China

$\mathrm{Tel} / \mathrm{Fax}+86-10-84322148$

Email yangzhiyun2016@163.com
Background and Aims: Although antiviral treatment has been shown to reduce mortality in hepatitis B virus (HBV)-related hepatocellular carcinoma (HCC) patients with high HBVDNA levels, it is still unclear whether it is useful in reducing mortality in patients with low HBV-DNA levels.

Methods: A retrospective analysis of $756 \mathrm{HBV}$-associated HCC patients at the Beijing Ditan Hospital with HBV-DNA levels $<500 \mathrm{IU} / \mathrm{mL}$ was conducted between January 2008 and June 2017. Patients were divided into antiviral and non-antiviral groups based on whether they received nucleos(t)ide analogue (NA) treatment when they were diagnosed with HCC in our hospital for the first time. We used 1:4 frequency matching by age, gender, tumor size, Barcelona Clinic Liver Cancer (BCLC) staging, anti-tumor therapy, cirrhosis, diabetes, and hyperlipoidemia to compare the antiviral $(n=366)$ and non-antiviral $(n=100)$ groups. A Cox multivariate regression analysis was employed to evaluate the effects of NA therapy on the hazard ratio (HR), and the Kaplan-Meier survival curve was used to determine the mortality risk in patients with HCC. A Log rank test was performed to analyze the effects of NA therapy on the survival rate of patients with HCC.

Results: After propensity score matching, the 1-, 3-, and 5-year overall survival (OS) rates for the antiviral and non-antiviral groups were $82.5 \%, 68.6 \%$, and $52.2 \%$, and $61.0 \%, 51.0 \%$, and $38.0 \%$, respectively. The l-year progression-free survival (PFS) rates for the two groups were $68.0 \%$ and $47.0 \%$, respectively. The OS of the antiviral group was significantly higher than that of the control group $(\mathrm{P}<0.001, \mathrm{P}=0.001$, and $\mathrm{P}=0.013$, respectively). The 1-year PFS for the antiviral group was also significantly better than that for the non-antiviral groups $(\mathrm{P}=0.005)$. After adjusting for confounding prognostic factors in the Cox model, the HR of 5-year death after antiviral treatment was 0.721 (95\% confidence interval [CI], 0.530-0.980, $\mathrm{P}=0.037$ ). Antiviral therapy is an independent protective factor for 5-year mortality in patients with $\mathrm{HCC}$ and low-level viremia.

Conclusion: Antiviral therapy significantly reduced mortality in HCC patients with low HBV-DNA levels.

Keywords: antiviral therapy, hepatitis B virus, low-level viremia, hepatocellular carcinoma, prognosis, nucleos(t)ide analog

\section{Introduction}

Primary liver cancer is the sixth most commonly diagnosed cancer and the third leading cause of cancer-related deaths worldwide. ${ }^{1}$ Hepatocellular carcinoma (HCC) is the dominant type of liver cancer. Hepatitis B virus (HBV) is the leading cause of incident cases of liver cancer and deaths worldwide, accounting for approximately $33 \%{ }^{2}$ 
Although there are several HCC treatment strategies, such as liver resection (LR), radiofrequency ablation (RFA), transarterial chemoembolization (TACE), or transplantation, as first-line therapy, the prognosis of HCC remains poor. ${ }^{3}$

In addition to anti-tumor treatment, antiviral therapy may benefit patients with HBV-associated HCC. Several studies have reported that antiviral therapy including TACE and RFA is minimally invasive, can improve the survival rate, and reduce the recurrence rate after resection. ${ }^{4-14}$ Therefore, it is recommended that HBVassociated HCC patients, particularly those with high viral loads, receive antiviral therapy. A retrospective cohort study recently revealed that $19.9 \%$ of chronic hepatitis $\mathrm{B}(\mathrm{CHB})$ patients had low-level viremia (LLV; 20$2000 \mathrm{IU} / \mathrm{mL}$ ) after entecavir (ETV) treatment. $^{15}$ Although patients with HBV-DNA levels of $<2000 \mathrm{IU} /$ $\mathrm{mL}$ are typically identified as inactive or low-risk HBV carriers, the postoperative tumor recurrence and mortality rates among these patients are still high. ${ }^{16,17}$ Therefore, inhibiting HBV DNA replication and improving the reduction of the virus in vivo is still important in $\mathrm{HCC}$ patients with LLV. However, it is unclear whether antiviral therapy can improve patient survival or reduce recurrence.

The Management of Hepatocellular Carcinoma guidelines issued by the European Association for the Study of the Liver 2018 did not recommend any antiviral treatment for HBV-associated HCC. There is little evidence that HCC patients can benefit from antiviral therapy, and antiviral treatment has no direct anti-tumor effect. The Hepatitis B Management Guidelines issued by EASL 2017 indicate that antiviral therapy should be provided to patients with compensated or decompensated cirrhosis, with detectable HBV-DNA levels. ${ }^{18}$ However, a recent study revealed that low-level viremia was associated with an increased risk of $\mathrm{HCC}$, indicating that LLV is not harmless. $^{20}$ Increasing evidence shows that antiviral therapy with entecavir, tenofovir disoproxil, and tenofovir alafenamide can reduce the incidence of HCC in HBsAgpositive patients regardless of HBV-DNA levels. ${ }^{21-29}$ However, it remains unclear whether HCC patients with low HBV-DNA levels can be effectively treated with antiviral therapy. This study was conducted to investigate this question.

We evaluated the role of antiviral therapy in $\mathrm{HCC}$ patients with LLV. The assessment of nucleos(t)ide analog (NA) therapy-attributable mortality and recurrence using propensity score matching (PSM) analysis was conducted to reduce heterogeneity between groups for further analysis. The potential prognostic characteristics of OS and PFS were also investigated.

\section{Methods}

\section{Study Design and Participants}

This retrospective cohort study was conducted to test the efficacy of antiviral therapy in HBV-associated HCC patients with low HBV-DNA levels and was approved by the Beijing Ditan Hospital. Between January 2008 and June 2017, 756 patients with HBV-DNA levels < 500 $\mathrm{IU} / \mathrm{mL}$ were newly diagnosed with HCC. Based on the 2017 guideline approved by the European Association for the Study of the Liver (EASL), lamivudine (LAM), telbivudine (TBV), entecavir (ETV), adefovir dipivoxil (ADV), tenofovir disoproxil fumarate (TDF), and tenofovir alafenamide (TAF) were administered as the antiviral drugs. ${ }^{18}$ Patients in the antiviral group received NAs, whereas those in the non-antiviral group did not receive any antiviral treatment. Until there was unacceptable toxicity or consent was withdrawn, antiviral treatment was continued. The study was designed and driven by researchers and was not supported by any pharmaceutical company.

Patients who were newly diagnosed with HBVassociated HCC between January 2008 and June 2017 were eligible for enrollment. HCC was diagnosed based on criteria provided by the European Association for the Study of the Liver. ${ }^{19}$

The inclusion criteria for this study were (1) age 18 to 75 years, (2) radiologic diagnosis of $\mathrm{HCC}^{19}{ }^{19}$ (3) positive test for hepatitis B surface antigen (HBsAg), (4) initial serum HBV-DNA level $<500 \mathrm{IU} / \mathrm{mL}$, and (5) good renal function (serum creatinine level $<133 \mathrm{mmol} / \mathrm{L}$ ).

The exclusion criteria were (1) positive tests for antibodies against the hepatitis $\mathrm{C}$ virus (HCV-Ab) and human immunodeficiency virus (HIV); (2) previous treatment of HCC; (3) complicated with other malignancies; and (4) treated with best support care.

\section{Diagnosis and Treatments for HCC}

The diagnosis and staging of HCC were established according to criteria provided by the European Association for the Study of the Liver. ${ }^{19}$ LR, RFA, and TACE are the initial treatment methods for HCC. After the initial treatment with LR, RFA, or TACE, patients who were eligible for subsequent treatments received RFA, TACE, and/or sorafenib as required. The subsequent 
treatments depended on tumor burden, liver function, and patient requirements. After LR, RFA, or TACE for 6-8 weeks, patients whose residual tumor enhancement and vascularity could be observed on CT imaging or hepatic artery angiography were recommended to repeat TACE or RFA combined with sorafenib therapy. Patients who were not suitable for any follow-up treatment were recommended for sorafenib treatment. Liver transplantation was not performed in our institute; therefore, it was not used as a follow-up treatment for any patient in this study. The follow-up ended on June 31, 2017.

\section{Antiviral Therapy}

We collected information about patients who received NAs. According to the EASL Clinical Practice Guidelines, ${ }^{18}$ we recommended that every patient with chronic hepatitis B receives antiviral treatment before or after LR, TACE, or RFA regardless of the presence of cirrhosis. During our study, lamivudine, adefovir, telbivudine, and entecavir were the available treatment options in China. However, patients were allowed to make choices and decisions (including receiving NAs and the type of NAs) according to their socioeconomic status. The patients treated with NA therapy $(n=715)$ were initially treated with lamivudine, adefovir, telbivudine, or entecavir. Patients were recommended to use alternative or additional NAs when HBV resistance was diagnosed. Among these, patients who were initially treated with lamivudine or telbivudine were administered adefovir as an additional NA (tenofovir was not available during the study period at our institute). Patients initially treated with adefovir were switched to entecavir.

\section{Statistical Analysis}

Continuous data are expressed as mean \pm standard deviation. Non-normally distributed data are expressed as medians with ranges. Serum HBV-DNA levels are expressed logarithmically. The social science statistical program (SPSS 22.0) and R statistical software (version 4.1.0) was used for statistical analysis. The Student's $t$-test or Mann-Whitney nonparametric $U$-test was used to compare quantitative values. Categorical variables were tested using the chi-square test or Fisher's exact test. PSM was performed to reduce heterogeneity between the antiviral and non-antiviral groups. The propensity score was estimated based on the tumor size and Barcelona clinical liver cancer (BCLC) staging. The Kaplan-Meier method was used to calculate overall survival (OS) and progression-free survival (PFS), and the Log rank test was used to compare the differences. The Cox proportional hazard regression model was used to gradually select variables for univariate and multivariate analyses. Univariate and multivariate Cox analysis (backward stepwise, maximum likelihood ratio method) were used to elucidate independent risk factors affecting 1-year or 5-year survival of patients. Statistical significance was defined as a P-value $<0.05$.

\section{Results}

\section{Patients' Characteristics}

As shown in Figure 1, from January 2008 to June 2017, 2111 patients were diagnosed with $\mathrm{HBV}$-associated HCC at the Beijing Ditan Hospital, Beijing, China, and 853 patients exhibited HBV-DNA levels $<500 \mathrm{IU} / \mathrm{mL}$.

The number of patients with $\mathrm{HBV}$-associated $\mathrm{HCC}$ enrolled in this study according to the inclusion and exclusion criteria was 756, including 583 men and 173 women. The age range was $45-67$ years, with a median age of 55 years. The NA treatment and control groups were determined based on whether the patient received NA antiviral therapy. Table 1 presents the differences between the two groups in the baseline data of hyperlipoidemia, cirrhosis, HBeAg level, white blood cell count (WBC), platelet (PLT), and alanine aminotransferase (ALT) levels $(\mathrm{P}<0.05)$.

\section{Survival Analysis Before and After PSM Processing}

As shown in Figure 2, before PSM, the 1-year OS rates of the antiviral treatment and control groups were $84.9 \%$ and 69.8\%, respectively, and the Log rank test difference was statistically significant $(\mathrm{P}<0.001)$. The 1 -year PFS rates of the two groups were $67.7 \%$ and $51.9 \%$, respectively, and the Log rank test was statistically different $(\mathrm{P}=$ 0.0013). As shown in Figure 2, the 5-year OS rates of the antiviral treatment and control groups were $37.7 \%$ and $32.1 \%$, respectively, and the Log rank test difference was statistically significant $(\mathrm{P}=0.0016)$. The PFS rates of the two groups were $20.0 \%$ and $11.3 \%$, respectively, and the Log rank test was statistically different $(P=0.016)$. All patients were followed up for 1 year or 5 years, and the OS and PFS rates of the patients in the antiviral treatment group were superior to those of the patients in the control group.

As shown in Table 2, after 1:4 frequency matching by age, gender, tumor size, Barcelona Clinic Liver Cancer 


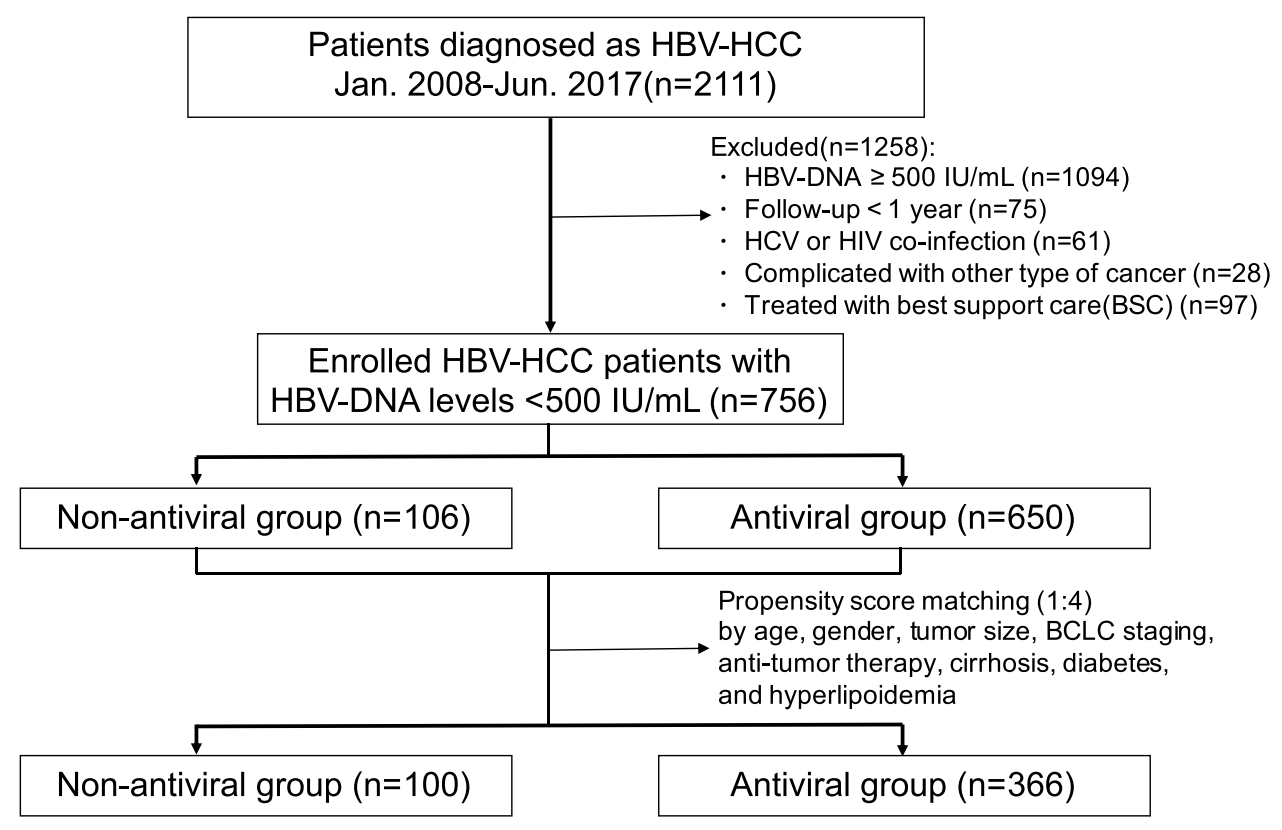

Figure I Flow chart of patient enrollment.

(BCLC) staging, anti-tumor therapy, cirrhosis, diabetes, and hyperlipoidemia of the antiviral treatment and control groups, the 1-, 3-, and 5-year OS rates were $82.5 \%, 68.6 \%$, and $52.2 \%$, and $61.0 \%, 51.0 \%$, and $38.0 \%$, respectively. All values exhibited statistical differences $(\mathrm{P}<0.05)$, and the survival rate of the antiviral treatment group was higher than that of the control group. The 1-year PFS rates of the two groups were $68.0 \%$ and $47.0 \%$, respectively. The 1-year PFS for the antiviral group was also significantly better than that for the non-antiviral groups $(\mathrm{P}$ $=0.005)$.

\section{Univariate and Multivariate Analyses for I-Year OS After PSM Processing}

Univariate Cox analysis revealed that antiviral therapy, $\gamma$ glutamyl transferase ( $\gamma$-GGT) level, MELD score, alphafetoprotein (AFP) level, tumor size, and BCLC stage were significantly associated with 1-year OS (Table 3).

NA therapy was determined to be an independent protective factor for 1 -year OS (hazard ratio $[\mathrm{HR}]=0.564$, $95 \%$ confidence interval $[\mathrm{CI}] 0.382-0.833, \mathrm{P}=0.004)$, and $\gamma$-GGT $(\geq 60 \mathrm{U} / \mathrm{L}, \mathrm{P}<0.001)$, MELD group (MELD score $\geq 6.3, \mathrm{P}<0.001$ ), AFP ( $\geq 400 \mathrm{ng} / \mathrm{mL}, \mathrm{P}=0.008)$, tumor size $(\geq 5 \mathrm{~cm}, \mathrm{P}<0.001)$, and BCLC stage $(\mathrm{C}-\mathrm{D}, \mathrm{P}<$ $0.001)$ were identified as independent risk factors for 1 -year OS using multivariate analysis.

\section{Univariate and Multivariate Analyses for 5-Year OS After PSM Processing}

Univariate Cox analysis revealed that antiviral therapy, anti-tumor therapy, MELD score, AFP, tumor size, and BCLC stage were significantly associated with 5-year OS (Table 4).

NA therapy was determined to be an independent protective factor for 5-year OS $(\mathrm{HR}=0.721,95 \%$ CI 0.530 $0.980, \mathrm{P}=0.037$ ), and anti-tumor therapy (Minimally invasive or Systemic therapy, $\mathrm{P}<0.05)$, MELD group (MELD score $\geq 6.3, \mathrm{P}<0.001)$, AFP $(\geq 400 \mathrm{ng} / \mathrm{mL}, \mathrm{P}<$ 0.001 ), tumor size ( $\geq 5 \mathrm{~cm}, \mathrm{P}<0.001$ ), and BCLC stage $(\mathrm{C}-\mathrm{D}, \mathrm{P}=0.027)$ were identified as independent risk factors for 5-year OS by multivariate analysis.

\section{Subgroup Analysis for I-Year PFS After PSM Processing}

The PFS meantime of the antiviral group was 10.79 months, significantly longer than that of the control group (5.92 months, HR 0.575; 95\% CI 0.41-0.807, P = 0.001). The association between PFS and all baseline stratification factors was analyzed using the Cox proportional hazards model. This analysis demonstrated that the most significant benefit of NA therapy was observed in patients with the following characteristics: age $\geq 50$ years, male sex, without diabetes or hyperlipoidemia, presence of cirrhosis, PLT $\leq 100 * 10^{\wedge} 9 / \mathrm{L}$, ALT $<50 \mathrm{IU} / \mathrm{L}$, tumor size $\geq$ 
Table I Demographic and Clinical Characteristics of Study Patients

\begin{tabular}{|c|c|c|c|}
\hline & Non-Antiviral Group, $\mathrm{n}=106(\%)$ & Antiviral Group, $n=650(\%)$ & $P$ value \\
\hline Age, years (mean $\pm S D$ ) & $56.5(50.0,63.0)$ & $56.0(50.0,63.0)$ & 0.745 \\
\hline Gender (Male) & $80(75.5 \%)$ & $503(77.4 \%)$ & 0.664 \\
\hline Diabetes & $21(19.8 \%)$ & $147(22.6 \%)$ & 0.520 \\
\hline Hyperlipoidemia & $12(\mid 1.3 \%)$ & $36(5.5 \%)$ & $0.024 *$ \\
\hline Cirrhosis (Yes) & $89(84.0 \%)$ & $605(93.1 \%)$ & $0.002 *$ \\
\hline $\mathrm{HBeAg}, \mathrm{S} / \mathrm{CO}$ & $0.40(0.33,0.53)$ & $0.44(0.34,1.16)$ & $0.01^{*}$ \\
\hline WBC, $10^{9} / \mathrm{L}$ & $5.54(3.80,7.30)$ & $4.25(2.96,5.6 I)$ & $<0.00 I^{*}$ \\
\hline Hemoglobin, $g / L$ & $132.2(|| 7.3,|4| .4)$ & $133.4(|| 5.0, \mid 46.3)$ & 0.356 \\
\hline Platelets, $10^{9} / \mathrm{L}$ & 125.6(80.5, I68.4) & $89.4(58.9,138.9)$ & $<0.001 *$ \\
\hline Albumin, $g / L$ & $39.1(33.2,42.9)$ & $38.6(34.0,41.8)$ & 0.726 \\
\hline Total bilirubin, umol/L & $16.7(10.7,37.4)$ & $16.6(\mid I .5,23.8)$ & 0.632 \\
\hline ALT, IU/L & $32.8(21.0,53.6)$ & $26.2(19.5,36.1)$ & $0.001 *$ \\
\hline Creatinine, $\mu \mathrm{mol} / \mathrm{L}$ & $66.6(56.0,80.0)$ & $66.9(57.0,77.1)$ & 0.918 \\
\hline Prothrombin activity, \% & $81.7(68.4,95.8)$ & $81.7(69.8,92.0)$ & 0.739 \\
\hline$\alpha$-Fetoprotein, $\mathrm{ng} / \mathrm{mL}$ & $10.5(3.5,77.1)$ & $10.9(4.0,84.9)$ & 0.975 \\
\hline Tumor size, cm & & & 0.715 \\
\hline$<5$ & $70(66.0 \%)$ & $458(70.5 \%)$ & \\
\hline$>5$ & $36(34.0 \%)$ & $192(29.5 \%)$ & \\
\hline Tumor multiplicity (solitary) & $74(69.8 \%)$ & $436(67.1 \%)$ & 0.577 \\
\hline BCLC staging & & & 0.191 \\
\hline $0-A$ & $52(49.1 \%)$ & $353(54.3 \%)$ & \\
\hline B & $29(27.4 \%)$ & $195(30.0 \%)$ & \\
\hline C & $15(\mid 4.2 \%)$ & $69(10.6 \%)$ & \\
\hline $\mathrm{D}$ & $10(9.4 \%)$ & $33(5.1 \%)$ & \\
\hline Anti-tumor therapy & & & 0.966 \\
\hline Resection & $12(\mid 1.3 \%)$ & $77(6.2 \%)$ & \\
\hline Minimally invasive & $88(83.0 \%)$ & $533(82.0 \%)$ & \\
\hline Systemic therapy & $6(5.7 \%)$ & $40(6.2 \%)$ & \\
\hline
\end{tabular}

Note: $*_{p}<0.05$

Abbreviations: $\mathrm{HBeAg}$, hepatitis B early antigen; WBC, white blood cell; ALT, alanine aminotransferase; BCLC, Barcelona Clinic Liver Cancer.

$5 \mathrm{~cm}, \mathrm{BCLC}$ grade $0-\mathrm{A}$ and $\mathrm{C}$, and minimally invasive (Figure 3).

\section{Subgroup Analysis for I- and 5-Year OS After PSM Processing}

The 1-year OS meantime of the antiviral group was 12.00 months, significantly longer than that of the control group (12.00 months, HR 0.408 ; 95\% CI $0.274-0.608$, P < $0.001)$. The association between 1-year OS and all baseline stratification factors was analyzed using the Cox proportional hazards model. This analysis demonstrated that the significant benefit of opting for NA therapy was observed in patients with the following characteristics: age $\geq 50$ years, without diabetes or hyperlipoidemia, presence of cirrhosis, BCLC grade 0 - C, minimally invasive, and systemic therapy (Figure 4).
The 5-year OS meantime of the antiviral group was 32.58 months, significantly longer than that of the control group (21.09 months, HR $0.681 ; 95 \%$ CI $0.51-0.911)$. The association between 5 -year OS and all baseline stratification factors was analyzed using the Cox proportional hazards model. This analysis demonstrated that the significant benefit of NA therapy was observed in patients with the following characteristics: age $\geq 50$ years, male sex, without diabetes or hyperlipoidemia, the presence of cirrhosis, $\mathrm{HBeAg}<1 \mathrm{~S} / \mathrm{CO}, \mathrm{PLT} \leq 100 * 10^{\wedge} 9 / \mathrm{L}, \mathrm{AFP} \geq 8.8$ $\mathrm{ng} / \mathrm{mL}$, tumor size $\geq 5 \mathrm{~cm}$, and BCLC grade 0 A (Figure 5).

\section{Discussion}

At present, the lower limit of detection of serum HBV DNA is generally $300-1000$ copies $/ \mathrm{mL}$ or $20-100 \mathrm{IU} / \mathrm{mL}$. 
A
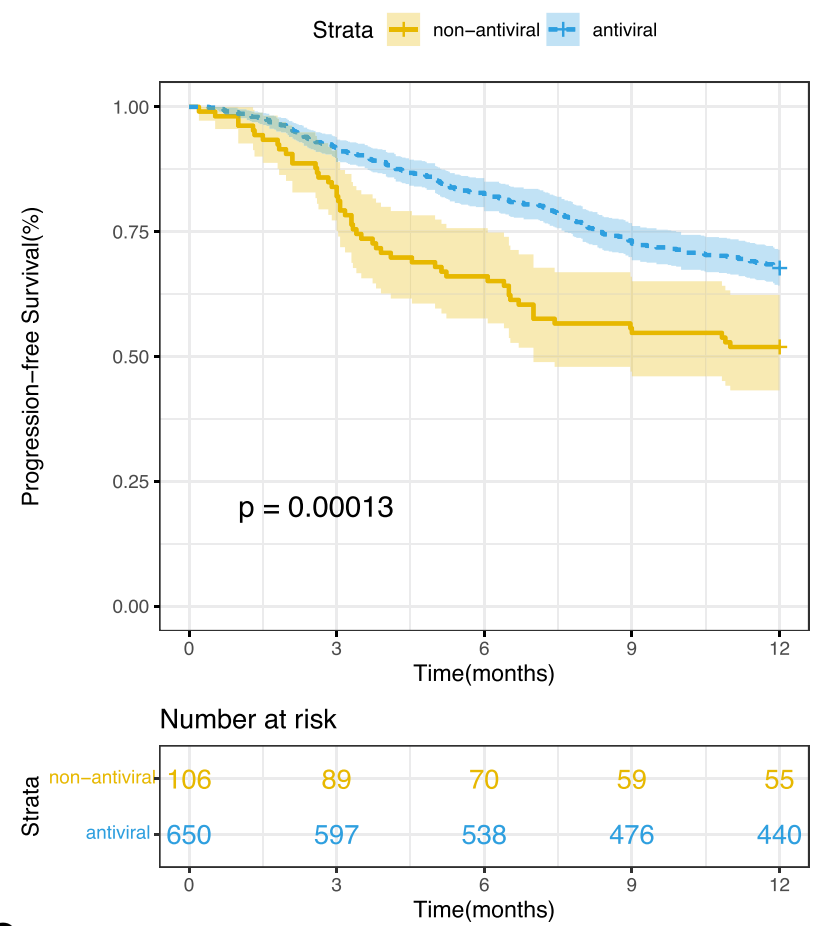

C
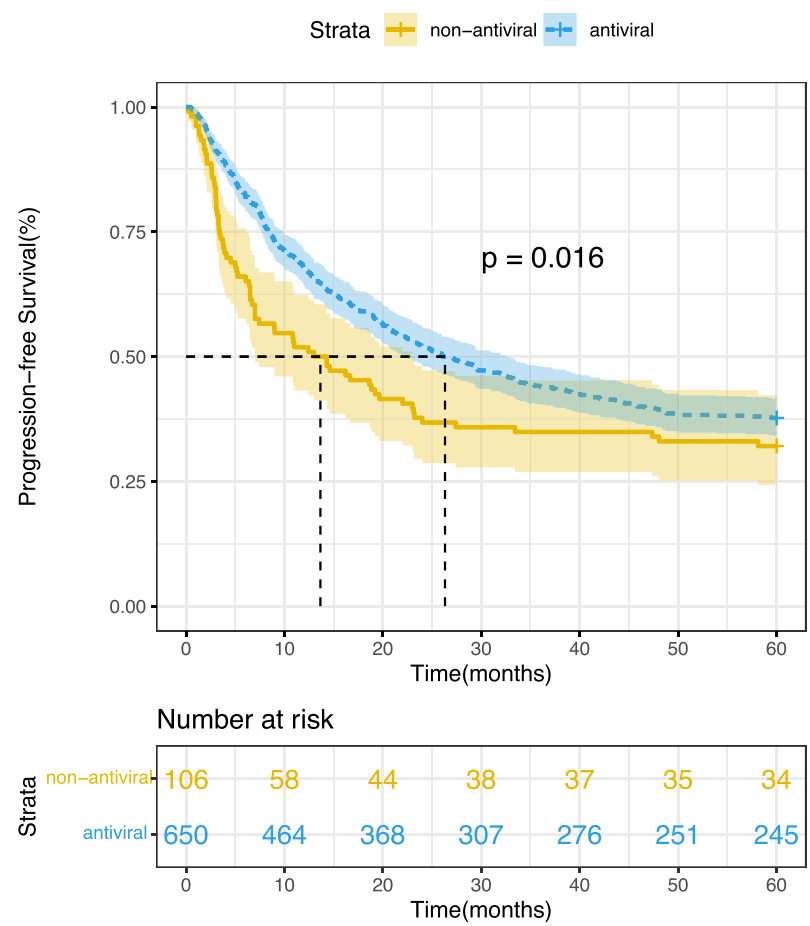

B
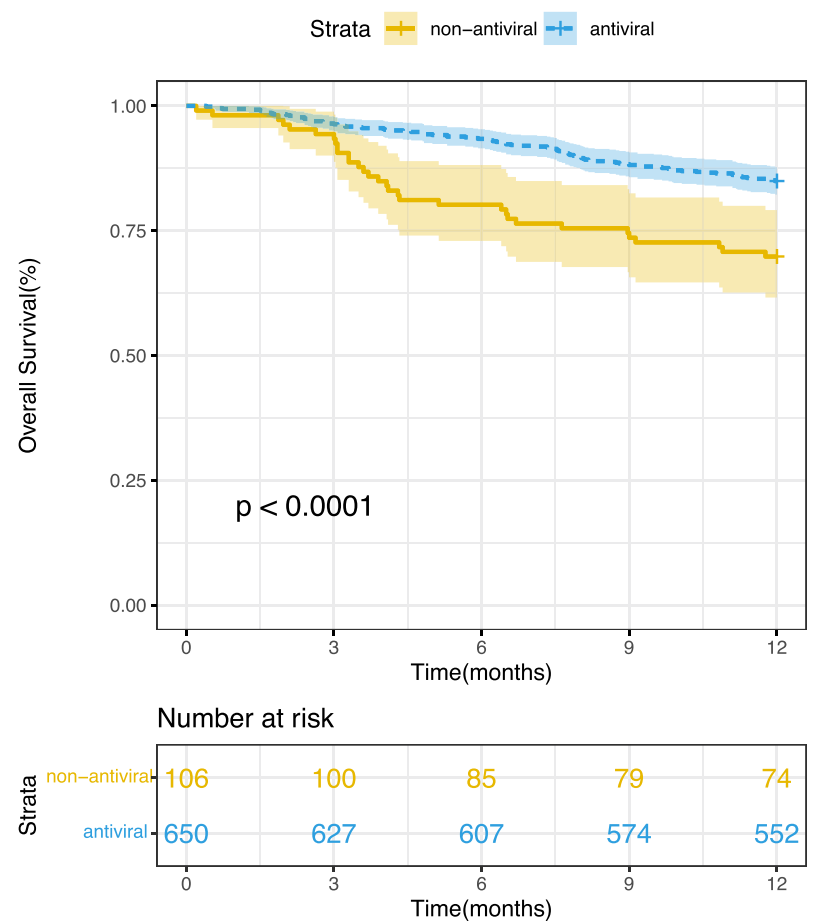

D
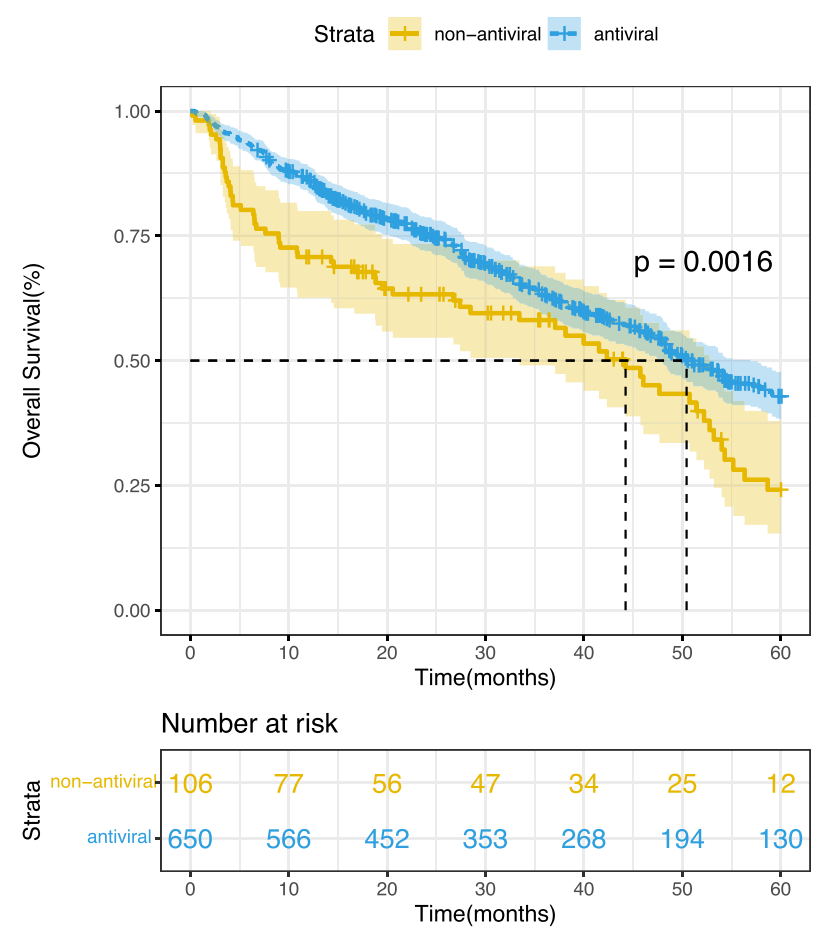

Figure 2 Kaplan-Meier curves of overall survival (OS) and progression-free survival (PFS) of 756 patients. (A) OS ( $<<0.00 \mathrm{I})$ and $(\mathbf{B})$ PFS $(\mathrm{P}=0.00 \mathrm{I})$ for I year, and (C) OS $(P=0.005)$ and $(D)$ PFS $(P<0.00 I)$ for 5 years. 
Table 2 Baseline Characteristics of the Study Patients After the I:4 Propensity Score Analysis

\begin{tabular}{|c|c|c|c|}
\hline & Non-Antiviral Group, $n=100(\%)$ & Antiviral Group, $n=366(\%)$ & $P$ value \\
\hline Age, years & $57(50,63)$ & $55(49,62)$ & 0.520 \\
\hline Gender (Male) & $76(76.0 \%)$ & $28 I(76.8 \%)$ & 0.871 \\
\hline Diabetes & $19(19.0 \%)$ & $59(16.1 \%)$ & 0.494 \\
\hline Hyperlipoidemia & $9(9.0 \%)$ & $21(5.7 \%)$ & 0.239 \\
\hline Cirrhosis (Yes) & $87(87.0 \%)$ & $330(90.2 \%)$ & 0.361 \\
\hline $\mathrm{HBeAg}, \mathrm{S} / \mathrm{CO}$ & $0.42(0.35,0.48)$ & $0.42(0.35,0.76)$ & 0.112 \\
\hline WBC, $10^{9} / \mathrm{L}$ & $5.49(4.04,7.33)$ & $4.14(2.90,5.57)$ & $<0.001 *$ \\
\hline Hemoglobin, g/L & $131.2(|14.0| 4 \mid .2)$, & $129.4(108.7, \mid 45.0)$ & 0.728 \\
\hline Platelets, $10^{9} / \mathrm{L}$ & $126.0(83.4,177.0)$ & $85.2(56.3,139.7)$ & $<0.001 *$ \\
\hline Albumin, g/L & $37.9(32.4,42.5)$ & $37.5(32.0,40.9)$ & 0.244 \\
\hline Total bilirubin, umol/L & $17.4(10.8,37.3)$ & $12.1(17.9,29.1)$ & 0.788 \\
\hline ALT, IU/L & $33.8(21.0,53.4)$ & $26.7(19.8,37.1)$ & $0.002^{*}$ \\
\hline Creatinine, $\mu \mathrm{mol} / \mathrm{L}$ & $66.0(55.7,76.0)$ & $66.0(58.0,77.0)$ & 0.869 \\
\hline Prothrombin activity, \% & $79.7(63.5,94.4)$ & $79.0(65.2,91.0)$ & 0.904 \\
\hline$\alpha$-Fetoprotein, $\mathrm{ng} / \mathrm{mL}$ & $14.6(3.6,108.2)$ & $12.6(4.6,124.8)$ & 0.907 \\
\hline Tumor size, $\mathrm{cm}$ & & & 0.465 \\
\hline$<5$ & $68(68.0 \%)$ & $219(59.8 \%)$ & \\
\hline$>5$ & $32(32.0 \%)$ & $147(40.2 \%)$ & \\
\hline Tumor multiplicity (solitary) & $65(65.0 \%)$ & $232(63.4 \%)$ & 0.766 \\
\hline BCLC staging & & & 0.988 \\
\hline $0-A$ & $50(50.0 \%)$ & $184(50.3 \%)$ & \\
\hline B & $28(28.0 \%)$ & $105(28.7 \%)$ & \\
\hline C & $13(13.0 \%)$ & $48(13.1 \%)$ & \\
\hline $\mathrm{D}$ & $9(9.0 \%)$ & $29(7.9 \%)$ & \\
\hline Anti-tumor therapy & & & 0.881 \\
\hline Resection & $12(12.0 \%)$ & $45(12.3 \%)$ & \\
\hline Minimally invasive & $84(84.0 \%)$ & $302(82.5 \%)$ & \\
\hline Systemic therapy & $4(4.0 \%)$ & $19(5.2 \%)$ & \\
\hline \multicolumn{4}{|l|}{ Progress } \\
\hline I year & $47(47.0 \%)$ & $117(32.0 \%)$ & $0.005^{*}$ \\
\hline \multicolumn{4}{|l|}{ Death } \\
\hline I year & $39(39.0 \%)$ & $64(17.5 \%)$ & $<0.001 *$ \\
\hline 3 year & $49(49.0 \%)$ & $115(31.4 \%)$ & $0.001 *$ \\
\hline 5 year & $62(62.0 \%)$ & $175(47.8 \%)$ & $0.013^{*}$ \\
\hline
\end{tabular}

Note: ${ }^{*} p<0.05$.

Abbreviations: $\mathrm{HBeAg}$, hepatitis B early antigen; WBC, white blood cell; ALT, alanine aminotransferase; BCLC, Barcelona Clinic Liver Cancer.

The serum HBV-DNA level of the patient is not equal to the viral load in the patient's body fluids or liver.

Although serum HBV DNA cannot be detected in the vast majority of $\mathrm{HBs} A g$-positive patients, the replication of the virus has not stopped. A considerable number of patients, particularly those with HCC development, have sustained HBV transcription and reverse transcription activities in the liver. Other steps including viral transcription, translation, and further downstream events are not inhibited, as reflected by the continuous detection of viral proteins and nucleic acids, such as HBV pre-genomic RNA (pgRNA), covalently closed circular DNA (cccDNA), HBsAg, and other viral biomarkers, in the serum and liver. $^{30-32}$ Therefore, in HBV-related HCC patients, although the serum virus level is low, persistent HBV infection may be an important factor for tumor recurrence and abnormal liver function. ${ }^{8}$ In addition, patients undergoing HCC anti-tumor therapy such as LR or TACE may develop HBV reactivation. ${ }^{33,34}$ Although novel compounds are emerging, current therapeutic options are still limited to 
Table 3 Univariate and Multivariate Cox Hazards Analysis for I-Year Overall Survival of All Patients with Hepatocellular Carcinoma

\begin{tabular}{|c|c|c|c|c|c|c|}
\hline & \multicolumn{3}{|c|}{ Univariate Cox Analysis } & \multicolumn{3}{|c|}{ Multivariate Cox Analysis } \\
\hline & $\mathbf{P}$ & HR & $95 \% \mathrm{Cl}$ & $\boldsymbol{P}$ & AdjustHR & $95 \% \mathrm{Cl}$ \\
\hline Gender (Male vs Female) & 0.947 & 1.013 & {$\left[\begin{array}{ll}0.691 & 1.486\end{array}\right]$} & & & \\
\hline Age, years ( $\geq 50$ vs $<50$ ) & 0.726 & 0.936 & {$\left[\begin{array}{lll}0.647 & 1.354\end{array}\right]$} & & & \\
\hline History of smoking (yes vs no) & 0.585 & 1.118 & {$\left[\begin{array}{lll}0.749 & 1.670\end{array}\right]$} & & & \\
\hline Alcohol abuse (yes vs no) & $0.046^{*}$ & 1.504 & {$\left[\begin{array}{lll}1.006 & 2.248\end{array}\right]$} & & & \\
\hline Family history of $\mathrm{CHB}$ (yes vs no) & 0.434 & 1.144 & {$\left[\begin{array}{lll}0.817 & 1.60 I\end{array}\right]$} & & & \\
\hline Family history of HCC (yes vs no) & 0.897 & 0.943 & {$\left[\begin{array}{lll}0.386 & 2.300\end{array}\right]$} & & & \\
\hline Diabetes (yes vs no) & 0.549 & 1.122 & {$\left[\begin{array}{lll}0.770 & 1.634\end{array}\right]$} & & & \\
\hline Hyperlipoidemia (yes vs no) & 0.311 & 1.358 & {$\left[\begin{array}{lll}0.752 & 2.453\end{array}\right]$} & & & \\
\hline Cirrhosis (yes vs no) & 0.055 & 2.226 & {$\left[\begin{array}{ll}0.983 & 5.038\end{array}\right]$} & & & \\
\hline WBC, $10^{9} / \mathrm{L}(\geq 4$ vs $<4)$ & 0.063 & 1.391 & {$\left[\begin{array}{ll}0.983 & 1.968\end{array}\right]$} & & & \\
\hline Platelets, $10^{9} / \mathrm{L}(\geq 100$ vs $<100)$ & 0.579 & 1.095 & {$\left[\begin{array}{lll}0.794 & \mathrm{I} .5 \mathrm{I} 2\end{array}\right]$} & & & \\
\hline ALT, IU/L ( $\geq 50$ vs $<50)$ & $0.86 \mathrm{I}$ & 0.964 & {$\left[\begin{array}{lll}0.636 & 1.459\end{array}\right]$} & & & \\
\hline $\mathrm{HBeAg}, \mathrm{S} / \mathrm{CO}(\geq \mathrm{I}$ vs $<1)$ & 0.579 & 0.940 & {$\left[\begin{array}{lll}0.755 & 1.170\end{array}\right]$} & & & \\
\hline Anti-tumor therapy & 0.219 & & & & & \\
\hline Minimally invasive vs Resection & 0.082 & 1.729 & {$\left[\begin{array}{lll}0.934 & 3.200\end{array}\right]$} & & & \\
\hline Systemic therapy vs Resection & 0.251 & 1.674 & {$\left[\begin{array}{llll}0.694 & 4.04 I\end{array}\right]$} & & & \\
\hline Antiviral(yes vs no) & $0.000 *$ & 0.474 & {$\left[\begin{array}{lll}0.327 & 0.687\end{array}\right]$} & $0.004^{*}$ & 0.564 & {$\left[\begin{array}{lll}0.382 & 0.833\end{array}\right]$} \\
\hline$\gamma$-GGT, IU/L $(\geq 60$ vs $<60)$ & $0.000 *$ & 3.524 & [2.484 4.999] & $0.000^{*}$ & 2.568 & {$\left[\begin{array}{lll}1.726 & 3.82 I\end{array}\right]$} \\
\hline MELD score $(\geq 6.3$ vs $<6.3)$ & $0.003 *$ & 1.693 & {$\left[\begin{array}{lll}1.198 & 2.393\end{array}\right]$} & $0.000 *$ & 1.990 & {$\left[\begin{array}{ll}1.360 & 2.912\end{array}\right]$} \\
\hline$\alpha$-Fetoprotein, $\mathrm{ng} / \mathrm{mL}(\geq 400$ vs $<400)$ & $0.000 *$ & 2.575 & {$\left[\begin{array}{lll}1.840 & 3.604\end{array}\right]$} & $0.008^{*}$ & 1.675 & {$\left[\begin{array}{ll}1.143 & 2.455\end{array}\right]$} \\
\hline Tumor Size, $\mathrm{cm}(\geq 5$ vs $<5)$ & $0.000 *$ & 5.102 & {$[3.5857 .26 \mathrm{I}]$} & $0.000^{*}$ & 2.709 & {$\left[\begin{array}{l}1.817 \\
4.040]\end{array}\right.$} \\
\hline $\mathrm{BCLC}$ staging (C-D vs 0-B) & $0.000 *$ & 3.131 & {$[2.1974 .462]$} & $0.000 *$ & 2.082 & {$\left[\begin{array}{lll}1.379 & 3.142\end{array}\right]$} \\
\hline
\end{tabular}

Note: *p $<0.05$.

Abbreviations: CHB, chronic hepatitis B; HCC, hepatocellular carcinoma; WBC, white blood cell; ALT, alanine aminotransferase; HBeAg, hepatitis B early antigen; $\gamma$-GGT, $\gamma$-glutamyl transpeptidase; MELD, model for end-stage liver disease; BCLC, Barcelona Clinic Liver Cancer.

NAs and pegylated interferon (PEG-IFN). NAs are well tolerated and can effectively suppress HBV replication. ${ }^{35}$ Long-term NA treatment induced marked depletion of cccDNA in the majority of patients. ${ }^{36}$ Antiviral therapy can reduce $\mathrm{HBV}$ postoperative reactivation in $\mathrm{HCC}$ patients with preoperative HBV-DNA levels $<100 \mathrm{IU} / \mathrm{mL}^{37}$ Therefore, the NA that inhibits HBV DNA replication and increases the reduction of the virus in vivo is still important in HCC patients with low viral loads.

The present results showed that in HBV-associated HCC patients with low viral loads, antiviral therapy significantly reduced the recurrence rate of the tumor in patients after 1 year or even 5 years after matching the main tumor factors of the antiviral and control groups. This was consistent with the findings of previously reported HBV-associated HCC patients with high HBV levels, exhibiting a high degree of consistency. ${ }^{4}$ Furthermore, NA therapy significantly enhanced not only 1-year PFS but also 1- and 5-year OS in patients with HCC, particularly those with normal ALT. Antiviral therapy can reverse liver dysfunction and normalize ALT levels in patients with HBV-associated HCC. ${ }^{38}$ Our study has revealed that antiviral therapy can improve the prognosis of patients with HCC although the viral loads are low and liver function is normal. Therefore, regardless of HBV-DNA and ALT levels, antiviral treatment is crucial in patients with HBV-associated HCC.

Active replication of HBV initiates the development of liver cancer through both direct and indirect carcinogenesis. ${ }^{39-44}$ Persistent viremia has been shown to impair tumor immune surveillance leading to viral replication and hepatocellular carcinoma development. ${ }^{45-47}$ A recent study proposed that HBV-pgRNA in HBVassociated HCC patients with undetectable serum HBVDNA predicts the response to NA treatment more sensitively and promotes proliferation, stemness, and tumorigenicity of HCC cells. Mechanistically, pgRNA could upregulate the expression of IGF2BP3, a well-proven oncoprotein, at the post-transcriptional level. ${ }^{48}$ These patients are more likely to develop multicentric cancer in the liver and exhibit 
Table 4 Univariate and Multivariate Cox Analysis for 5-Year Overall Survival of All Patients with Hepatocellular Carcinoma

\begin{tabular}{|c|c|c|c|c|c|c|}
\hline & \multicolumn{3}{|c|}{ Univariate Cox Analysis } & \multicolumn{3}{|c|}{ Multivariate Cox Analysis } \\
\hline & $\boldsymbol{P}$ & HR & $95 \% \mathrm{Cl}$ & $\boldsymbol{P}$ & AdjustHR & $95 \% \mathrm{Cl}$ \\
\hline Gender (Male vs Female) & 0.488 & 1.098 & {$\left[\begin{array}{lll}0.844 & \mathrm{I} .427\end{array}\right]$} & & & \\
\hline Age, years ( $\geq 50$ vs $<50$ ) & 0.077 & 1.272 & {$\left[\begin{array}{lll}0.974 & 1.662\end{array}\right]$} & & & \\
\hline History of smoking (yes vs no) & 0.344 & 1.136 & {$\left[\begin{array}{lll}0.872 & 1.480\end{array}\right]$} & & & \\
\hline Alcohol abuse (yes vs no) & 0.489 & 1.1 & {$\left[\begin{array}{ll}0.839 & 1.442\end{array}\right]$} & & & \\
\hline Family history of $\mathrm{CHB}$ (yes vs no) & 0.725 & 0.958 & {$\left[\begin{array}{ll}0.753 & 1.219\end{array}\right]$} & & & \\
\hline Family history of HCC (yes vs no) & 0.564 & 1.215 & {$\left[\begin{array}{ll}0.627 & 2.354\end{array}\right]$} & & & \\
\hline Diabetes (yes vs no) & 0.620 & 1.067 & {$\left[\begin{array}{lll}0.826 & 1.379\end{array}\right]$} & & & \\
\hline Hyperlipoidemia (yes vs no) & 0.060 & 0.631 & {$\left[\begin{array}{lll}0.390 & 1.019\end{array}\right]$} & & & \\
\hline Cirrhosis (yes vs no) & 0.525 & 1.227 & {$\left[\begin{array}{ll}0.653 & 2.303\end{array}\right]$} & & & \\
\hline WBC, $10^{9} / \mathrm{L}(\geq 4$ vs $<4)$ & 0.929 & 1.011 & {$\left[\begin{array}{lll}0.790 & 1.295\end{array}\right]$} & & & \\
\hline PLT, $10^{9} / \mathrm{L}(\geq 100$ vs $<100)$ & 0.267 & 1.157 & {$\left[\begin{array}{lll}0.894 & 1.497\end{array}\right]$} & & & \\
\hline ALT, IU/L ( $\geq 50$ vs <50) & 0.356 & 1.145 & {$\left[\begin{array}{lll}0.859 & 1.528\end{array}\right]$} & & & \\
\hline $\mathrm{HBeAg}, \mathrm{S} / \mathrm{CO}(\geq \mathrm{I}$ vs $<1)$ & 0.375 & 1.075 & {$\left[\begin{array}{lll}0.916 & 1.261\end{array}\right]$} & & & \\
\hline Anti-tumor therapy & $0.000 *$ & & & $0.000 *$ & & \\
\hline Minimally invasive vs Resection & $0.012^{*}$ & 2.102 & {$\left[\begin{array}{lll}1.173 & 3.764\end{array}\right]$} & $0.000^{*}$ & 2.851 & {$\left[\begin{array}{lll}1.753 & 4.637]\end{array}\right]$} \\
\hline Systemic therapy vs Resection & $0.000 *$ & 2.710 & 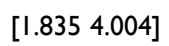 & $0.002^{*}$ & 2.861 & {$\left[\begin{array}{ll}1.453 & 5.634\end{array}\right]$} \\
\hline Antiviral(yes vs no) & $0.000 *$ & 0.586 & {$\left[\begin{array}{lll}0.440 & 0.779\end{array}\right]$} & $0.037^{*}$ & 0.721 & {$\left[\begin{array}{lll}0.530 & 0.980\end{array}\right]$} \\
\hline$\gamma$-GGT, IU/L $(\geq 60$ vs $<60)$ & $0.000 *$ & 1.886 & {$\left[\begin{array}{lll}1.5 & 12 & 2.353\end{array}\right]$} & & & \\
\hline MELD score $(\geq 6.3$ vs $<6.3)$ & $0.000 *$ & 1.682 & {$\left[\begin{array}{lll}1.342 & 2.108\end{array}\right]$} & $0.000 *$ & 1.667 & {$\left[\begin{array}{ll}1.273 & 2.182\end{array}\right]$} \\
\hline$\alpha$-Fetoprotein, $\mathrm{ng} / \mathrm{mL}(\geq 400$ vs $<400$ ) & $0.000 *$ & 2.011 & {$\left[\begin{array}{ll}1.563 & 2.587\end{array}\right]$} & $0.000 *$ & 1.732 & {$\left[\begin{array}{lll}1.281 & 2.34 I\end{array}\right]$} \\
\hline Tumor Size, $\mathrm{cm}(\geq 5$ vs $<5)$ & $0.000 *$ & 2.228 & {$\left[\begin{array}{ll}1.723 & 2.880\end{array}\right]$} & $0.000 *$ & 1.778 & {$\left[\begin{array}{ll}1.343 & 2.355\end{array}\right]$} \\
\hline BCLC staging (C-D vs $0-B$ ) & $0.000 *$ & 2.298 & {$\left[\begin{array}{lll}1.817 & 2.905\end{array}\right]$} & 0.027 & 1.396 & {$\left[\begin{array}{ll}1.040 & 1.875\end{array}\right]$} \\
\hline
\end{tabular}

Note: ${ }^{*}<<0.05$.

Abbreviations: CHB, chronic hepatitis B; HCC, hepatocellular carcinoma; WBC, white blood cell; ALT, alanine aminotransferase; HBeAg, hepatitis B early antigen; $\gamma$-GGT, $\gamma$-glutamyl transpeptidase; MELD, model for end-stage liver disease; BCLC, Barcelona Clinic Liver Cancer.

upregulation of adhesion molecules that are present on the cell wall of the hepatic sinus wall, thereby indicating enhanced tumor development.

Many tumor characteristics are irreversible in HBVassociated HCC patients. However, antiviral therapy can alter the status of HBV infection. The major therapeutic effects of antiviral therapy are to prevent HBV reactivation, inhibit hepatitis activity, reduce inflammation in the liver, reverse cirrhosis, and improve liver function. ${ }^{49}$ Antiviral therapy may reduce tumor recurrence by downregulating hepatic inflammation and related nuclear signaling pathways, leading to tumor transformation at the cellular level, reversing fibrosis, and reducing regenerative stimulation at the tissue level. ${ }^{36,50,51}$ Antiviral therapy also reduces the expression of hepatitis $\mathrm{B}$ virus $\mathrm{X}$ protein $(\mathrm{HBx})$, preventing HBV-DNA from integrating into the host chromosome or harnessing its malignant potential at the genomic level. ${ }^{52,53}$ Moreover, we observed that antiviral therapy significantly reduced the rate of tumor recurrence and improved the OS of the patients. Therefore, for HCC patients with low HBV-DNA levels, we recommend that the concept of antiviral therapy be changed from "rigid monitoring" to "the lower the better, the sooner the better."

There are a few limitations in the current study; some must be considered when interpreting the study findings. First, patients received variable NAs, and different timing and duration of antiviral treatment also affected HBV reactivation. Second, cirrhosis was defined by sonographic features that are qualitative and prone to variation between observers. Although the propensity score was matched, the median of serum white blood cells and platelets in the antiviral group was lower than that in the non-antiviral group (Table 2). More accurate markers of liver cirrhosis based preferably on noninvasive tests should be used to define cirrhosis status. Third, this was a single-institution study. Additional studies are required to better understand the effects of antiviral therapy on the prognosis of HCC in other ethnic or geographical regions. Finally, genotype data were not available. However, it is well known that HBV genotypes $\mathrm{B}$ and $\mathrm{C}$ are the most common in HBsAgpositive patients in the Asia Pacific region. ${ }^{54}$ 


\begin{tabular}{|c|c|c|c|c|c|c|}
\hline Subgroup & Non-antiviral group(\%) & Antiviral group(\%) & & & Hazard Ratio(95\%) & $P$ values \\
\hline All patients & $100(100 \%)$ & $366(100 \%)$ & $<$ & & $0.575(0.41-0.807)$ & 0.001 \\
\hline \multicolumn{7}{|l|}{ Age, years } \\
\hline$<50$ & $23(23.0 \%)$ & $90(24.6 \%)$ & ط- & & $0.729(0.331-1.604)$ & 0.432 \\
\hline$\geq 50$ & $77(77.0 \%)$ & $276(75.4 \%)$ & H늠 & & $0.548(0.376-0.799)$ & 0.002 \\
\hline \multicolumn{7}{|l|}{ Gender } \\
\hline Female & $24(24.0 \%)$ & $85(23.2 \%)$ & $\mapsto$ & & $0.53(0.251-1.119)$ & 0.096 \\
\hline Male & $76(76.0 \%)$ & $281(76.8 \%)$ & $H$ & & $0.583(0.398-0.852)$ & 0.005 \\
\hline \multicolumn{7}{|l|}{ Diabetes mellitus } \\
\hline No & $81(81.0 \%)$ & $307(83.9 \%)$ & HE- & & $0.532(0.366-0.773)$ & 0.001 \\
\hline Yes & 19(19.0\%) & $59(16.1 \%)$ & $\longmapsto$ & & $0.817(0.363-1.835)$ & 0.624 \\
\hline \multicolumn{7}{|l|}{ Hyperlipoidemia } \\
\hline No & $91(91.0 \%)$ & $345(94.3 \%)$ & $\mathrm{H}-\mathrm{H}$ & & $0.549(0.389-0.777)$ & 0.001 \\
\hline Yes & $9(9.0 \%)$ & $21(5.7 \%)$ & $\longrightarrow$ & & $0.84(0.154-4.587)$ & 0.84 \\
\hline \multicolumn{7}{|l|}{ Cirhosis } \\
\hline No & $13(13.0 \%)$ & $36(9.8 \%)$ & 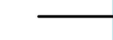 & - & $\Rightarrow \quad 1.391(0.295-6.552)$ & 0.677 \\
\hline Yes & $87(87.0 \%)$ & $330(90.2 \%)$ & $r$ 늘-1 & & $0.523(0.37-0.741)$ & $<0.001$ \\
\hline \multicolumn{7}{|l|}{ HBeAg, S/CO } \\
\hline$<1$ & $67(67.0 \%)$ & $220(60.6 \%)$ & H- & & $0.585(0.383-0.893)$ & 0.013 \\
\hline$\geq 1$ & $10(10 \%)$ & $74(20.2 \%)$ & $\mapsto$ & & $0.585(0.383-0.893)$ & 0.013 \\
\hline unknown & $23(23 \%)$ & $72(19.7 \%)$ & $\longmapsto$ & & $0.688(0.315-1.503)$ & 0.348 \\
\hline \multicolumn{7}{|l|}{ WBC, $10^{\wedge} 9 / \mathrm{L}$} \\
\hline$<4$ & $26(26.0 \%)$ & $168(45.9 \%)$ & $\mapsto$ & & $0.486(0.264-0.893)$ & 0.02 \\
\hline $4 \leq W B C<10$ & $70(70.0 \%)$ & $190(51.9 \%)$ & $\mapsto$ & & $0.58(0.379-0.887)$ & 0.012 \\
\hline$\geq 10$ & $4(4.0 \%)$ & $8(2.2 \%)$ & & & $2.776(0.323-23.88)$ & 0.352 \\
\hline \multicolumn{7}{|l|}{ PLT, 10^9/L } \\
\hline$\leq 100$ & $35(35.0 \%)$ & $209(57.1 \%)$ & $\mapsto-1$ & & $0.485(0.287-0.819)$ & 0.007 \\
\hline$>100$ & $65(65.0 \%)$ & $157(42.9 \%)$ & $\mapsto$ & & $0.658(0.418-1.034)$ & 0.07 \\
\hline \multicolumn{7}{|l|}{ ALT, IU/L } \\
\hline$<50$ & $71(71.0 \%)$ & $322(88.0 \%)$ & $\mapsto$ & & $0.6(0.405-0.888)$ & 0.011 \\
\hline$\geq 50$ & $29(29.0 \%)$ & $44(12.0 \%)$ & $\mapsto$ & & $0.527(0.251-1.107)$ & 0.091 \\
\hline \multicolumn{7}{|l|}{ MELD score } \\
\hline$<6.3$ & $66(66.0 \%)$ & $245(66.9 \%)$ & $\mapsto$ & & $0.618(0.39-0.98)$ & 0.041 \\
\hline$\geq 6.3$ & $34(34.0 \%)$ & $121(33.1 \%)$ & $\mapsto$ & & $0.516(0.312-0.852)$ & 0.01 \\
\hline \multicolumn{7}{|l|}{ AFP, $n g / m L$} \\
\hline$<8.8$ & $46(46.0 \%)$ & $175(47.8 \%)$ & $\mapsto$ & & $0.583(0.386-0.881)$ & 0.01 \\
\hline$\geq 8.8$ & $54(54.0 \%)$ & $191(52.2 \%)$ & $\mapsto$ & & $0.497(0.274-0.902)$ & 0.021 \\
\hline \multicolumn{7}{|l|}{ Tumor Size(cm) } \\
\hline$<5$ & $68(68.0 \%)$ & $219(59.8 \%)$ & $\mapsto$ & & $0.75(0.469-1.199)$ & 0.229 \\
\hline$\geq 5$ & $32(32.0 \%)$ & $147(40.2 \%)$ & 늘-1 & & $0.349(0.213-0.571)$ & $<0.001$ \\
\hline \multicolumn{7}{|l|}{ BCLC group } \\
\hline $0-A$ & $50(50.0 \%)$ & $184(50.3 \%)$ & $\mapsto$ & & $0.496(0.268-0.919)$ & 0.026 \\
\hline B & $28(28.0 \%)$ & $105(28.7 \%)$ & $\mapsto$ & & $0.568(0.312-1.304)$ & 0.064 \\
\hline C & $13(13.0 \%)$ & $48(13.1 \%)$ & $\mapsto$ & & $0.432(0.214-0.87)$ & 0.019 \\
\hline $\mathrm{D}$ & $9(9.0 \%)$ & $29(7.9 \%)$ & $\longrightarrow$ & & $0.821(0.321-2.102)$ & 0.681 \\
\hline \multicolumn{7}{|c|}{ Anti-tumor therapy } \\
\hline Resection & $12(12.0 \%)$ & $45(12.3 \%)$ & & & $\Rightarrow \quad 1.111(0.236-5.233)$ & 0.894 \\
\hline Minimally invasive & $84(84.0 \%)$ & $302(82.5 \%)$ & $\mathrm{H}-\mathrm{H}$ & & $0.572(0.4-0.818)$ & 0.002 \\
\hline Systemic therapy & $4(4.0 \%)$ & $19(5.2 \%)$ & $H$ & & $0.22(0.048-1.011)$ & 0.052 \\
\hline
\end{tabular}

Figure 3 Subgroup analysis for I-year progression-free survival after PSM processing. 


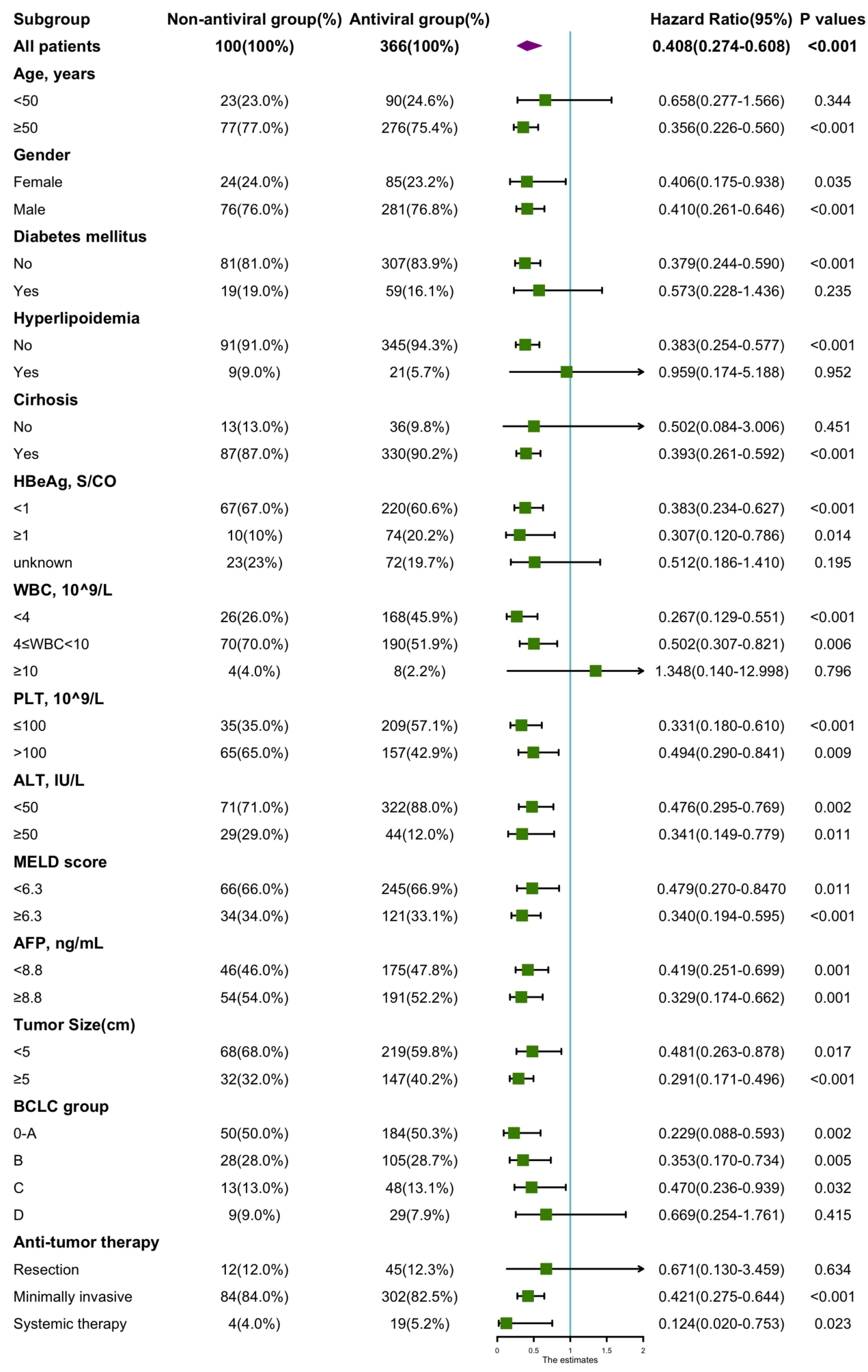

Figure 4 Subgroup analysis for I-year overall survival after PSM processing. 


\begin{tabular}{|c|c|c|c|c|c|c|}
\hline Subgroup & Non-antiviral group(\%) & Antiviral group(\%) & & & Hazard Ratio(95\%) & $P$ values \\
\hline All patients & $100(100 \%)$ & $366(100 \%)$ & $<$ & & $0.681(0.51-0.911)$ & 0.01 \\
\hline \multicolumn{7}{|l|}{ Age, years } \\
\hline$<50$ & $23(23.0 \%)$ & $90(24.6 \%)$ & •—- & $\longrightarrow$ & $0.639(0.324-1.258)$ & 0.195 \\
\hline$\geq 50$ & $77(77.0 \%)$ & $276(75.4 \%)$ & $\mathrm{H}-\mathrm{C}$ & & $0.707(0.513-0.976)$ & 0.035 \\
\hline \multicolumn{7}{|l|}{ Gender } \\
\hline Female & $24(24.0 \%)$ & $85(23.2 \%)$ & •-口 & $\rightarrow$ & $0.702(0.366-1.345)$ & 0.286 \\
\hline Male & $76(76.0 \%)$ & $281(76.8 \%)$ & H-1 & & $0.685(0.495-0.948)$ & 0.022 \\
\hline \multicolumn{7}{|l|}{ Diabetes mellitus } \\
\hline No & $81(81.0 \%)$ & $307(83.9 \%)$ & $\mapsto-1$ & & $0.655(0.475-0.903)$ & 0.01 \\
\hline Yes & $19(19.0 \%)$ & $59(16.1 \%)$ & - & & $0.816(0.412-1.614)$ & 0.558 \\
\hline \multicolumn{7}{|l|}{ Hyperlipoidemia } \\
\hline No & $91(91.0 \%)$ & $345(94.3 \%)$ & $\mathrm{H}-1$ & & $0.680(0.505-0.196)$ & 0.011 \\
\hline Yes & $9(9.0 \%)$ & $21(5.7 \%)$ & $\mapsto$ & -1 & $0.305(0.075-1.237)$ & 0.096 \\
\hline \multicolumn{7}{|l|}{ Cirhosis } \\
\hline No & $13(13.0 \%)$ & $36(9.8 \%)$ & - & $\longrightarrow$ & $\Rightarrow \quad 1.438(0.313-6.615)$ & 0.641 \\
\hline Yes & $87(87.0 \%)$ & $330(90.2 \%)$ & $\mathrm{H}-\mathrm{H}$ & & $0.651(0.484-0.875)$ & 0.004 \\
\hline \multicolumn{7}{|l|}{ HBeAg, S/CO } \\
\hline$<1$ & $67(67.0 \%)$ & $220(60.6 \%)$ & $\mapsto$ & & $0.68(0.477-0.97)$ & 0.033 \\
\hline$\geq 1$ & $10(10 \%)$ & $74(20.2 \%)$ & $\mapsto$ & & $0.492(0.228-1.064)$ & 0.071 \\
\hline unknown & $23(23 \%)$ & $72(19.7 \%)$ & $\longmapsto$ & & $0.829(0.404-1.701)$ & 0.61 \\
\hline \multicolumn{7}{|l|}{ WBC, $10^{\wedge} 9 / \mathrm{L}$} \\
\hline$<4$ & $26(26.0 \%)$ & $168(45.9 \%)$ & 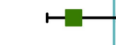 & & $0.622(0.374-1.034)$ & 0.067 \\
\hline $4 \leq W B C<10$ & $70(70.0 \%)$ & $190(51.9 \%)$ & $\mapsto$ & & $0.651(0.45-0.942)$ & 0.023 \\
\hline$\geq 10$ & $4(4.0 \%)$ & $8(2.2 \%)$ & & & $2.029(0.235-17.491)$ & 0.52 \\
\hline \multicolumn{7}{|l|}{ PLT, 10^9/L } \\
\hline$\leq 100$ & $35(35.0 \%)$ & $209(57.1 \%)$ & H-1 & & $0.565(0.37-0.862)$ & 0.008 \\
\hline$>100$ & $65(65.0 \%)$ & $157(42.9 \%)$ & •는 & $\rightarrow$ & $0.768(0.51-1.156)$ & 0.206 \\
\hline \multicolumn{7}{|l|}{ ALT, IU/L } \\
\hline$<50$ & $71(71.0 \%)$ & $322(88.0 \%)$ & $\mapsto$ & & $0.745(0.531-1.045)$ & 0.088 \\
\hline$\geq 50$ & $29(29.0 \%)$ & $44(12.0 \%)$ & $\mapsto$ & & $0.561(0.299-1.053)$ & 0.072 \\
\hline \multicolumn{7}{|l|}{ MELD score } \\
\hline$<6.3$ & $66(66.0 \%)$ & $245(66.9 \%)$ & $\mapsto$ & & $0.703(0.473-1.044)$ & 0.08 \\
\hline$\geq 6.3$ & $34(34.0 \%)$ & $121(33.1 \%)$ & $\mapsto$ & -1 & $0.721(0.469-1.108)$ & 0.135 \\
\hline \multicolumn{7}{|l|}{ AFP, ng/mL } \\
\hline$<8.8$ & $46(46.0 \%)$ & $175(47.8 \%)$ & H- & & $0.713(0.507-1.003)$ & 0.052 \\
\hline$\geq 8.8$ & $54(54.0 \%)$ & $191(52.2 \%)$ & $\mapsto$ & & $0.51(0.293-0.889)$ & 0.018 \\
\hline \multicolumn{7}{|l|}{ Tumor Size(cm) } \\
\hline$<5$ & $68(68.0 \%)$ & $219(59.8 \%)$ & $\mapsto$ & $\longrightarrow$ & $0.843(0.572-1.242)$ & 0.387 \\
\hline$\geq 5$ & $32(32.0 \%)$ & $147(40.2 \%)$ & $H-1$ & & $0.451(0.291-0.699)$ & $<0.001$ \\
\hline \multicolumn{7}{|l|}{ BCLC group } \\
\hline $0-A$ & $50(50.0 \%)$ & $184(50.3 \%)$ & $\mapsto$ & & $0.536(0.325-0.882)$ & 0.014 \\
\hline B & $28(28.0 \%)$ & $105(28.7 \%)$ & $\mapsto$ & & $0.666(0.391-1.134)$ & 0.135 \\
\hline C & $13(13.0 \%)$ & $48(13.1 \%)$ & & & $1.277(0.66-2.471)$ & 0.468 \\
\hline $\mathrm{D}$ & $9(9.0 \%)$ & $29(7.9 \%)$ & $\longrightarrow$ & 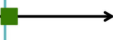 & $1.039(0.482-2.241)$ & 0.923 \\
\hline \multicolumn{7}{|c|}{ Anti-tumor therapy } \\
\hline Resection & $12(12.0 \%)$ & $45(12.3 \%)$ & Hen- & & $0.723(0.53-0.985)$ & 0.04 \\
\hline Minimally invasive & $84(84.0 \%)$ & $302(82.5 \%)$ & H- & & $0.723(0.53-0.985)$ & 0.04 \\
\hline Systemic therapy & $4(4.0 \%)$ & $19(5.2 \%)$ & $\mathrm{H} \longrightarrow$ & & $0.233(0.066-0.817)$ & 0.023 \\
\hline
\end{tabular}

Figure 5 Subgroup analysis for 5-year overall survival after PSM processing. 
In conclusion, prophylactic oral antiviral therapy reduced HCC recurrence and increased treatment efficacy in patients with low HBV-DNA levels. This ultimately improved the survival rate of patients with HBV-related HCC. This study suggests that patients with low serum HBV-DNA load should immediately receive potent antiviral therapy.

\section{Ethics Approval and Consent to Participate}

This study is in accordance with the Declaration of Helsinki and has been approved by the ethical committee of Beijing Ditan Hospital, Capital Medical University. The study is a retrospective cohort research, which only collect the clinical data of patients, do not interfere with the treatment plan of patients, and will not bring risks to the physiology of patients. The researchers will try their best to protect the information provided by patients from disclosing personal privacy. Retrospective study does not need to obtain informed consent, mainly because: (1) we only collect clinical laboratory data of patients, the risk is controllable; (2) some patients were out of contact or have died, and can not track their own signature of informed consent.

\section{Acknowledgments}

This study was supported by the Beijing Hospitals Authority' Ascent Plan (grant no. DFL20191803), National Science Foundation of China (grant no. 81874435), Special Fund of Capital Health Research and Development (grant no. 2020-2-2173), and Beijing Hospitals Authority Clinical Medicine Development of Special Funding Support (grant no. ZYLX202127).

\section{Author Contributions}

Authors made a significant contribution to this study. Conception and design of the study: Zhiyun Yang. Acquisition of data and follow-up: Xiaoli Liu, Lihua Yu, Fengna Yan. Statistical analysis: Xinhui Wang, Huiwen Yan, Dongdong Zhou. Writing - original draft: Xinhui Wang, Peng Wang. Approval of final version of the manuscript: all authors. All authors made a significant contribution to the work reported, whether that is in the conception, study design, execution, acquisition of data, analysis and interpretation, or in all these areas; took part in drafting, revising or critically reviewing the article; gave final approval of the version to be published; have agreed on the journal to which the article has been submitted; and agree to be accountable for all aspects of the work.

\section{Disclosure}

No potential conflict of interest relevant to this article was reported. The abstract of this paper was presented as a poster presentation at THE DIGITAL INTERNATIONAL LIVER CONGRESS, August 27-29, 2020 entitled Nucleos(t)ide Analogs Reduce Hepatocellular Carcinoma Mortality in Patients with Low HBV-DNA levels, reporting interim findings. The poster's abstract was published in "Poster Abstracts" in the Hyperlink: https://ilc-congress.eu/wp-content/uploads/ 2020/08/digital-ilc-2020-abstract-book-20-august.pdf.

\section{References}

1. Sung H, Ferlay J, Siegel RL, et al. Global cancer statistics 2020 : GLOBOCAN estimates of incidence and mortality worldwide for 36 cancers in 185 countries. CA Cancer J Clin. 2021;71:209-249. doi: $10.3322 /$ caac. 21660

2. Singal AG, Lampertico P, Nahon P. Epidemiology and surveillance for hepatocellular carcinoma: new trends. J Hepatol. 2020;72 (2):250-261. doi:10.1016/j.jhep.2019.08.025

3. Villanueva A, Longo DL. Hepatocellular carcinoma. $N$ Engl $J$ Med. 2019;380(15):1450-1462. doi:10.1056/NEJMra1713263

4. Huang G, Lau WY, Wang ZG, et al. Antiviral therapy improves postoperative survival in patients with hepatocellular carcinoma: a randomized controlled trial. Ann Surg. 2015;261(1):56-66. doi:10.1097/SLA.0000000000000858

5. Jang JW, Yoo SH, Nam HC, et al. Association of prophylactic anti-hepatitis B virus therapy with improved long-term survival in patients with hepatocellular carcinoma undergoing transarterial therapy. Clin Infect Dis. 2020;71(3):546-555. doi:10.1093/cid/ciz860

6. Sohn W, Kang TW, Choi SK, et al. Effect of oral antiviral treatment on long-term outcomes of radiofrequency ablation therapy for hepatitis B virus-related hepatocellular carcinoma. Oncotarget. 2016;7 (30):47794-47807. doi:10.18632/oncotarget.10026

7. Piao CY, Fujioka S, Iwasaki Y, et al. Lamivudine treatment in patients with HBV-related hepatocellular carcinoma-using an untreated, matched control cohort. Acta Med Okayama. 2005;59 (5):217-224

8. Kuzuya T, Katano Y, Kumada T, et al. Efficacy of antiviral therapy with lamivudine after initial treatment for hepatitis B virus-related hepatocellular carcinoma. $J$ Gastroenterol Hepatol. 2007;22 (11):1929-1935.

9. Kubo S, Tanaka H, Takemura S, et al. Effects of lamivudine on outcome after liver resection for hepatocellular carcinoma in patients with active replication of hepatitis B virus. Hepatol Res. 2007;37 (2):94-100.

10. Kim BK, Park JY, Kim DY, et al. Persistent hepatitis B viral replication affects recurrence of hepatocellular carcinoma after curative resection. Liver Int. 2008;28(3):393-401. doi:10.1111/j.14783231.2007.01625.x

11. Chan AC, Chok KS, Yuen WK, et al. Impact of antiviral therapy on the survival of patients after major hepatectomy for hepatitis B virus-related hepatocellular carcinoma. Arch Surg. 2011;146 (6):675-681. doi:10.1001/archsurg.2011.125 
12. Wu CY, Chen YJ, Ho HJ, et al. Association between nucleoside analogues and risk of hepatitis B virus-related hepatocellular carcinoma recurrence following liver resection. JAMA. 2012;308 (18):1906-1914. doi:10.1001/2012.jama.11975

13. Hann HW, Coben R, Brown D, et al. A long-term study of the effects of antiviral therapy on survival of patients with HBV-associated hepatocellular carcinoma (HCC) following local tumor ablation. Cancer Med. 2014;3(2):390-396. doi:10.1002/cam4.197

14. Hann HW, Bergin D, Coben R, DiMarino AJ. Prevention of new hepatocellular carcinoma with concomitant antiviral therapy in chronic hepatitis B patients whose initial tumor was successfully ablated. Int $J$ Cancer. 2011;128(3):739-742. doi:10.1002/ ijc. 25382

15. Ogawa E, Nomura H, Nakamuta $\mathrm{M}$, et al. Tenofovir alafenamide after switching from entecavir or nucleos(t)ide combination therapy for patients with chronic hepatitis B. Liver Int. 2020;40 (7):1578-1589. doi:10.1111/liv.14482

16. Huang G, Lau WY, Zhou WP, et al. Prediction of hepatocellular carcinoma recurrence in patients with low hepatitis $\mathrm{B}$ virus DNA levels and high preoperative hepatitis B surface antigen levels. JAMA Surg. 2014;149(6):519-527. doi:10.1001/jamasurg.2013.4648

17. Kim TS, Sinn DH, Kang W, et al. Hepatitis B virus DNA levels and overall survival in hepatitis B-related hepatocellular carcinoma patients with low-level viremia. J Gastroenterol Hepatol. 2019;34 (11):2028-2035. doi:10.1111/jgh.14750

18. Lampertico P, Agarwal K, Berg T. EASL 2017 clinical practice guidelines on the management of hepatitis B virus infection. J Hepatol. 2017;67(2):370-398. doi:10.1016/j.jhep.2017.03.021

19. European Association for the Study of the Liver. EASL clinical practice guidelines: management of hepatocellular carcinoma. J Hepatol. 2018;69(1):182-236.

20. Kim JH, Sinn DH, Kang W, et al. Low-level viremia and the increased risk of hepatocellular carcinoma in patients receiving entecavir treatment. Hepatology. 2017;66(2):335-343. doi:10.1002/ hep. 28916

21. Wu CY, Lin JT, Ho HJ, et al. Association of nucleos(t)ide analogue therapy with reduced risk of hepatocellular carcinoma in patients with chronic hepatitis B: a nationwide cohort study. Gastroenterology. 2014;147(1):143-51.e5. doi:10.1053/j. gastro.2014.03.048

22. Watanabe T, Tokumoto Y, Joko K, et al. Effects of long-term entecavir treatment on the incidence of hepatocellular carcinoma in chronic hepatitis B patients. Hepatol Int. 2016;10(2):320-327. doi:10.1007/s12072-015-9647-8

23. Papatheodoridis GV, Idilman R, Dalekos GN, et al. The risk of hepatocellular carcinoma decreases after the first 5 years of entecavir or tenofovir in Caucasians with chronic hepatitis B. Hepatology. 2017;66(5):1444-1453. doi:10.1002/hep.29320

24. Nguyen MH, Yang HI, Le A, et al. Reduced incidence of hepatocellular carcinoma in cirrhotic and noncirrhotic patients with chronic hepatitis B treated with tenofovir-a propensity score-matched study. J Infect Dis. 2019;219(1):10-18. doi:10.1093/infdis/jiy391

25. Yin GQ, Li J, Zhong B, Yang YF, Wang MR. New therapeutic options for persistent low-level viremia in patients with chronic hepatitis B virus infection: increase of entecavir dosage. World J Gastroenterol. 2021;27(8):666-676. doi:10.3748/wjg.v27. i8.666

26. Liaw YF, Sung JJ, Chow WC, et al. Lamivudine for patients with chronic hepatitis B and advanced liver disease. $N$ Engl J Med. 2004;351(15):1521-1531. doi:10.1056/NEJMoa033364

27. Eun JR, Lee HJ, Kim TN, Lee KS. Risk assessment for the development of hepatocellular carcinoma: according to on-treatment viral response during long-term lamivudine therapy in hepatitis B virus-related liver disease. J Hepatol. 2010;53(1):118-125. doi:10.1016/j.jhep.2010.02.026
28. Hosaka T, Suzuki F, Kobayashi M, et al. Long-term entecavir treatment reduces hepatocellular carcinoma incidence in patients with hepatitis B virus infection. Hepatology. 2013;58(1):98-107. doi:10.1002/hep. 26180

29. Kim WR, Berg T, Loomba R. Long term Tenofovir Disoproxil Fumarate (TDF) therapy and the risk of hepatocellular carcinoma. J Hepatol. 2013;58:S19. doi:10.1016/S0168-8278(13)60045-8

30. Mak LY, Huang Q, Wong DK, et al. Residual HBV DNA and pgRNA viraemia is associated with hepatocellular carcinoma in chronic hepatitis B patients on antiviral therapy. $J$ Gastroenterol. 2021;56 (5):479-488. doi:10.1007/s00535-021-01780-5

31. Hsu CW, Chu YD, Lai MW, et al. Hepatitis B Virus Covalently Closed Circular DNA Predicts Postoperative Liver Cancer Metastasis Independent of Virological Suppression. Cancers (Basel). 2021;13(3):538. doi:10.3390/cancers 13030538

32. Lam YF, Seto WK, Wong D, et al. Seven-year treatment outcome of entecavir in a real-world cohort: effects on clinical parameters, HBsAg and HBcrAg levels. Clin Transl Gastroenterol. 2017;8(10): e125. doi:10.1038/ctg.2017.51

33. Huang G, Li PP, Lau WY, et al. Antiviral therapy reduces hepatocellular carcinoma recurrence in patients with low HBV-DNA levels: a randomized controlled trial. Ann Surg. 2018;268(6):943-954. doi:10.1097/SLA.0000000000002727

34. Jang JW, Choi JY, Bae SH, et al. Transarterial chemo-lipiodolization can reactivate hepatitis $\mathrm{B}$ virus replication in patients with hepatocellular carcinoma. J Hepatol. 2004;41(3):427-435. doi:10.1016/j. jhep.2004.05.014

35. Buti M, Riveiro-Barciela M, Esteban R. Long-term safety and efficacy of nucleo(t)side analogue therapy in hepatitis B. Liver Int. 2018;38(Suppl 1):84-89. doi:10.1111/liv.13641

36. Lai CL, Wong D, Ip P, et al. Reduction of covalently closed circular DNA with long-term nucleos(t)ide analogue treatment in chronic hepatitis B. J Hepatol. 2017;66(2):275-281. doi:10.1016/j. jhep.2016.08.022

37. Xu M, Zhou Z, Xu R, Zhang H, Lin N, Zhong Y. Antiviral therapy predicts the outcomes following resection of hepatocellular carcinoma in patients negative for HBV DNA: a propensity score matching analysis. World J Surg Oncol. 2019;17(1):45. doi:10.1186/ s12957-019-1577-9

38. Lao XM, Luo G, Ye LT, et al. Effects of antiviral therapy on hepatitis $\mathrm{B}$ virus reactivation and liver function after resection or chemoembolization for hepatocellular carcinoma. Liver Int. 2013;33 (4):595-604. doi:10.1111/liv.12112

39. Kim CM, Koike K, Saito I, Miyamura T, Jay G. HBx gene of hepatitis B virus induces liver cancer in transgenic mice. Nature. 1991;351(6324):317-320. doi:10.1038/351317a0

40. Fallot G, Neuveut C, Buendia MA. Diverse roles of hepatitis B virus in liver cancer. Curr Opin Virol. 2012;2(4):467-473. doi:10.1016/j. coviro.2012.05.008

41. Levrero M, Zucman-Rossi J. Mechanisms of HBV-induced hepatocellular carcinoma. J Hepatol. 2016;64(1):S84-s101. doi:10.1016/j. jhep.2016.02.021

42. Kostyusheva A, Kostyushev D, Brezgin S, Volchkova E, Chulanov V. Clinical implications of hepatitis B virus RNA and covalently closed circular DNA in monitoring patients with chronic hepatitis B today with a gaze into the future: the field is unprepared for a sterilizing cure. Genes (Basel). 2018;9(10):483. doi:10.3390/genes9100483

43. Song F, Wei M, Wang J, et al. Hepatitis B virus-regulated growth of liver cancer cells occurs through the microRNA-340-5p-activating transcription factor 7-heat shock protein A member 1B axis. Cancer Sci. 2019;110(5):1633-1643. doi:10.1111/cas.14004

44. Wungu CDK, Ariyanto FC, Prabowo GI, Soetjipto S, Handajani R. Meta-analysis: association between hepatitis B virus preS mutation and hepatocellular carcinoma risk. J Viral Hepat. 2021;28(1):61-71. doi:10.1111/jvh.13402 
45. Chen Y, Tian Z. HBV-induced immune imbalance in the development of HCC. Front Immunol. 2019;10:2048. doi:10.3389/ fimmu.2019.02048

46. Sartorius K, Swadling L, An P, et al. The multiple roles of hepatitis $B$ virus $\mathrm{X}$ protein $(\mathrm{HBx})$ dysregulated microRNA in hepatitis $\mathrm{B}$ virus-associated hepatocellular carcinoma (HBV-HCC) and immune pathways. Viruses. 2020;12(7):746. doi:10.3390/v12070746

47. Hao X, Chen Y, Bai L, Wei H, Sun R, Tian Z. HBsAg-specific CD8 $(+) \mathrm{T}$ cells as an indispensable trigger to induce murine hepatocellular carcinoma. Cell Mol Immunol. 2021;18(1):128-137. doi:10.1038/ s41423-019-0330-1

48. Ding WB, Wang MC, Yu J, et al. HBV-pgRNA increases the stemness and promotes the development of HBV-related HCC through reciprocal regulation with IGF2BP3. Hepatology. 2021;74:1480-1495. doi:10.1002/hep.31850

49. Fanning GC, Zoulim F, Hou J, Bertoletti A. Therapeutic strategies for hepatitis B virus infection: towards a cure. Nat Rev Drug Discov. 2019;18(11):827-844. doi:10.1038/s41573-019-0037-0

50. Butler EK, Gersch J, McNamara A, et al. Hepatitis B virus serum DNA andRNA levels in nucleos(t)ide analog-treated or untreated patients during chronic and acute infection. Hepatology. 2018;68 (6):2106-2117. doi:10.1002/hep.30082
51. Liu S, Liu Z, Li W, et al. Factors associated with the biphasic kinetics of serum HBV RNA in patients with HBeAg-positive chronic hepatitis B treated with nucleos(t)ide analogues. Aliment Pharmacol Ther. 2020;52(4):692-700. doi:10.1111/apt.15890

52. Jin XL, Hong SK, Kim H, et al. Antiviral therapy may decrease HBx, affecting cccDNA and MSL2 in hepatocarcinogenesis. Oncol Lett. 2019;18(5):4984-4991.

53. Tak E, Hwang S, Lee HC, et al. Apoptosis of hepatitis B virus-expressing liver tumor cells induced by a high concentration of nucleos(t)ide analogue. Anticancer Res. 2016;36(11):6059-6069. doi:10.21873/anticanres.11195

54. Kurbanov F, Tanaka Y, Mizokami M. Geographical and genetic diversity of the human hepatitis B virus. Hepatol Res. 2010;40 (1):14-30.

\section{Publish your work in this journal}

The Journal of Hepatocellular Carcinoma is an international, peerreviewed, open access journal that offers a platform for the dissemination and study of clinical, translational and basic research findings in this rapidly developing field. Development in areas including, but not limited to, epidemiology, vaccination, hepatitis therapy, pathology and molecular tumor classification and prognostication are all considered for publication. The manuscript management system is completely online and includes a very quick and fair peer-review system, which is all easy to use. Visit http://www.dovepress.com/ testimonials.php to read real quotes from published authors. 